\title{
Synthesis and Thermal and Textural Characterization of Aniline Formaldehyde-Organoclay Composites
}

\author{
A. GÜrses ${ }^{a, *}$, Z. ERoĞLU ${ }^{a}, K \cdot$ GÜneŞ$^{a}$, Ç. DoĞAR ${ }^{b}$ \\ ${ }^{a}$ Atatürk University, Faculty of K.K. Education, 25240, Erzurum, Turkey \\ ${ }^{b}$ Erzincan University, Faculty of Education, 24030, Erzincan, Turkey
}

\begin{abstract}
In this study, the synthesis of aniline-formaldehyde resin, and its thermal and structural characterization, as well as the preparation and characterization of the resin-organoclay composites were carried out. For this, first, at $70^{\circ} \mathrm{C}$ and acidic conditions the aniline formaldehyde prepolymer was prepared and then cured at $120^{\circ} \mathrm{C}$ under vacuum. The structural and thermal characterization of the resin was made using FTIR and DSC techniques. By using the Cetyltrimethylammonium Bromide modified Montmorillonite (OMMT) and the synthesized resin, the resin-organoclay composites were prepared by melt intercalation method. Characterization of the resin-organoclay composites prepared with the different ratios of organoclay is made with the same techniques. The FTIR and thermal analysis results of the composites indicate that a cross-linked polymeric matrix was formed. The thermal behavior of the composites has also significantly changed compared to pure resin.
\end{abstract}

DOI: 10.12693/APhysPolA.129.853

PACS/topics: 81.70.Pg

\section{Introduction}

Formaldehyde resins represent a major class of polymers with a large variety of applications in the field of coating, mechanical, electrical and electronically industries, because of their good thermal, mechanical and electrical properties. These important properties are directly dependent on chemical structure of monomers used in synthesis. Generally, the precursor, such as phenol, substituted phenol, aromatic amine, polyphenol, cyclohexanone, urea, melamine, and aniline are used in the synthesis of active monomer by interacting with formaldehyde in the preparation of these polymers [1]. Aniline formaldehyde condensate (AFC) is a resinous polymer with amine $\left(-\mathrm{NH}_{2}\right)$ groups, which can be easily synthesized by polymerization of aniline in presence of formaldehyde in acidic medium [2]. The synthesis of polymeric resin containing reactive functional groups has been an active field of research in polymer science because it provides a great method for the modification of the polymers for the required application [3]. During the past decade, nanotechnology has become an active field of research because of its tremendous potential for a variety of applications. When the size of many established, well-studied materials is reduced to the nanoscale, radically improved or new surprising properties often emerge [4]. In recent years, polymer based nanocomposites reinforced with Na-Montmorillonite nanoclay particles have drawn significant attention. This is due to the fact that these types of composites show substantial improvements in mechanical, thermal and barrier properties over the pure polymer, and therefore, have

*corresponding author; e-mail: ahmetgu@yahoo.com potential in high-performance applications [5-12]. It is well known that nanoparticles can also enhance the mechanical properties and thermal degradation of nanocomposites. In general, the enhanced physical properties of polymer/nanocomposites are strongly dependent on the type (size, functionality, polarity, hydrophobicity, and acidity) and the amount of the organic modifiers used as treatments of the layered silicates nanofiller, such as Montmorillonite (MMT). Thus, the proper selection of modifier is necessary for substantial enhancement of polymer properties [13]. Organoclay produced by surface modifications of clay minerals with cationic surface active agents has received attention because it allows the creation of new materials nanocomposites and new applications [14].

The objective of this study is the synthesis of anilineformaldehyde resin, and its thermal and structural characterization, and also the preparation and characterization of the AF/organoclay composites.

\section{Materials and methods}

\subsection{Materials}

Aniline, and aqueous solution of formaldehyde (37\%), $\mathrm{NaOH}$, and $\mathrm{HCl}$ which were used in the synthesis of $\mathrm{AF}$ composites, were supplied from Merck. In order to prepare the organoclays, raw clay from Çankırı, Turkey was used. The cation exchange capacity (CEC) of clay was determined to be $147.9 \mathrm{meq} / 100 \mathrm{~g}$ by the methylene blue method (ANSI/ASTM C837-76) [15]. Cetyltrimethylammonium Bromide (CTAB) as a cationic surfactant, which was purchased from Merck, was used without further purification. The chemical composition of MMT, which has been determined by X-ray fluorescence spectrometry technique, is given in Table I. Typical properties of commercial long-chained hydrocarbon used in this study are shown in Table II. 
Chemical composition of the MMT.

\begin{tabular}{c|c|c|c|c|c|c|c|c|c}
\hline \hline \multicolumn{1}{c}{ Components [\%] } \\
\hline $\mathrm{SiO}_{2}$ & $\mathrm{Al}_{2} \mathrm{O}_{3}$ & $\mathrm{Fe}_{2} \mathrm{O}_{3}$ & $\mathrm{MgO}$ & $\mathrm{CaO}$ & $\mathrm{Na}_{2} \mathrm{O}$ & $\mathrm{K}_{2} \mathrm{O}$ & $\mathrm{TiO}_{3}$ & $\mathrm{SO}_{3}$ & Other \\
\hline 59.32 & 17.19 & 5.95 & 3.63 & 2.21 & 1.68 & 0.97 & 0.74 & 0.51 & 7.81
\end{tabular}

TABLE II

Some physical and chemical properties of long-chain hydrocarbon used in this study.

\begin{tabular}{c|c|c|c|c|c|c|c|c}
\hline \hline $\begin{array}{c}\text { Density } \\
\left(15^{\circ} \mathrm{C}\right) \\
{\left[\mathrm{kg} / \mathrm{m}^{3}\right]}\end{array}$ & $\begin{array}{c}\text { Calorific } \\
\text { value } \\
{[\mathrm{MJ} / \mathrm{kg}]}\end{array}$ & $\begin{array}{c}\text { Flash } \\
\text { point } \\
{\left[{ }^{\circ} \mathrm{C}\right]}\end{array}$ & $\begin{array}{c}\text { Water by } \\
\text { distillation, } \\
{[\text { wt.\%] }}\end{array}$ & $\mathrm{C}$ & $\mathrm{H}$ & $\mathrm{N}$ & $\mathrm{S}$ & Ash \\
\hline 990.7 & 42.74 & 105.8 & 0.1 & 83.4 & 11.9 & 0.8 & 1.5 & 0.03 \\
\hline
\end{tabular}

\subsection{Preparation of modified clay,} aniline-formaldehyde (AF) resin

and organo clay-aniline-formaldehyde composites

The combination of swelling and adsorption processes during intercalation and exfoliation of layered silicates was used in solution intercalation. The clay sample was purified by washing method, dried in a vacuum oven and sieved to give a $38-85 \mu \mathrm{m}$ size fraction using ASTM sieves. $0.3 \mathrm{~g}$ of long-chain hydrocarbon agent was dispersed in $500 \mathrm{ml}$ of CTAB aqueous solution $(240 \mathrm{mg} / \mathrm{l})$ for 30 minutes. After addition of $1.0 \mathrm{~g}$ of raw clay to the prepared dispersion, it was shaken at $200 \mathrm{rpm}$ shaking speed and at various temperatures (293, 313, and $333 \mathrm{~K}$ ), using a thermostatic shaker for 30 minutes. Finally, the mixture was filtered and, then dried in a vacuum oven at $383 \mathrm{~K}$ for 2 hours. The concentrations of $\mathrm{CTA}^{+}$in the supernatant after the adsorption were determined using a double-beam UV spectrophotometer at $375 \mathrm{~nm}$ accompanied with $0.02 \mathrm{ml}$ of $0.1 \%$ picric acid in $0.002 \mathrm{M} \mathrm{NaOH}$ and $0.40 \mathrm{ml}$ of 1,2-dichloroethane added per $1.0 \mathrm{ml}$ of the supernatant [16]. The calibration curve was found to be very reproducible and linear over the concentration range used in this study. The amount of the adsorbed CTAB was calculated from the concentrations in solution before and after adsorption. Blanks containing no CTAB were used for each series of experiments.

Aniline formaldehyde (AF) and the composites were prepared using in-situ intercalative polymerization method. The preparation procedure was as follows: aniline was mixed with formaldehyde (as a 37\% formalin solution) in acidic condition and, after adding a few drops of $40 \% \mathrm{NaOH}(\mathrm{pH}=9)$ in a glass reactor equipped with a mechanical stirrer, a thermometer and a reflux condenser and then synthesized organoclays in a given ratios were added to the mixture at $70^{\circ} \mathrm{C}$. Finally, the resultant mixture was dried under vacuum at $70^{\circ} \mathrm{C}$ for 1 hour.

The proposed reaction mechanism for the synthesis of aniline-formaldehyde resin is shown in Fig 1.

\subsection{Characterization of organo clay-aniline-formaldehyde composites}

Many techniques, such as FTIR, XRD, SEM and DSC have been employed for characterization of the composites in this study. XRD diffractograms for the raw clay

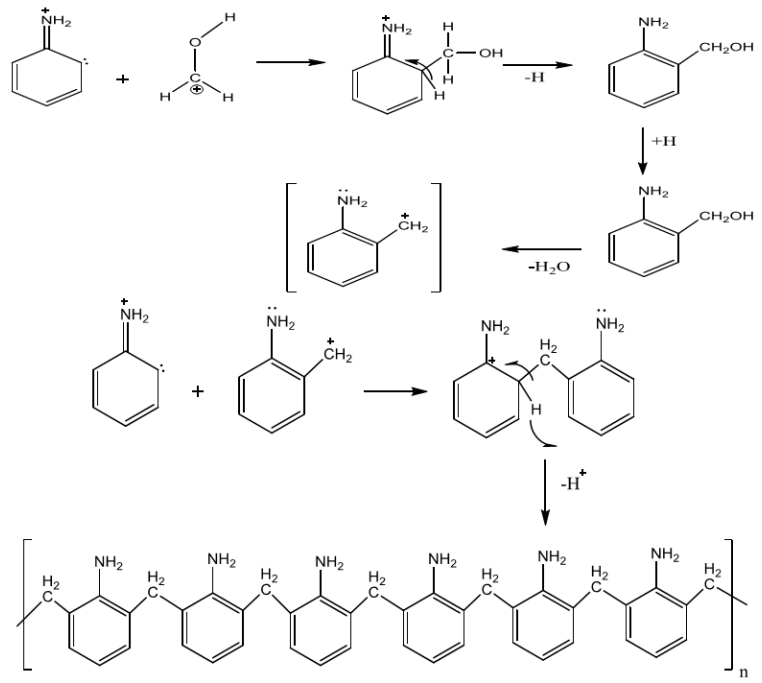

Fig. 1. A probable linear structure for AF resins.

and composites were taken in Bruker D8 Advance X-ray Diffractometer with $\mathrm{Cu} \mathrm{K}_{\alpha 1}(1.540 \AA)$ radiation, operating at $40 \mathrm{kV}$ and $30 \mathrm{~mA}$ over a $2 \theta$ range of $3-40^{\circ}$. The scanning rate of the instrument was $2 \%$ min. FTIR spectra for prepared pure composites were taken on a Perkin-Elmer Spectrum-One, by KBr pelleting method from 4000 to $400 \mathrm{~cm}^{-1}$. All measurements were obtained by averaging of 100 scans and at a resolution of $1 \mathrm{~cm}^{-1}$. The textural and morphological structures of the specimens were examined using a FEI-INSPECT S50 model scanning electron microscope (SEM) operating at $30 \mathrm{kV}$. The curing behaviors of the prepared composites were studied using a differential scanning calorimeter (DSC7020) under nitrogen atmosphere. A typical sample weight was about $10 \mathrm{mg}$, and the scan speed was $20^{\circ} \mathrm{C} / \mathrm{min}$.

\section{Results and discussion}

\subsection{FTIR analysis}

FTIR spectra for organoclay-reinforced AF composites containing various ratios of organoclay are given in Fig. 2 .

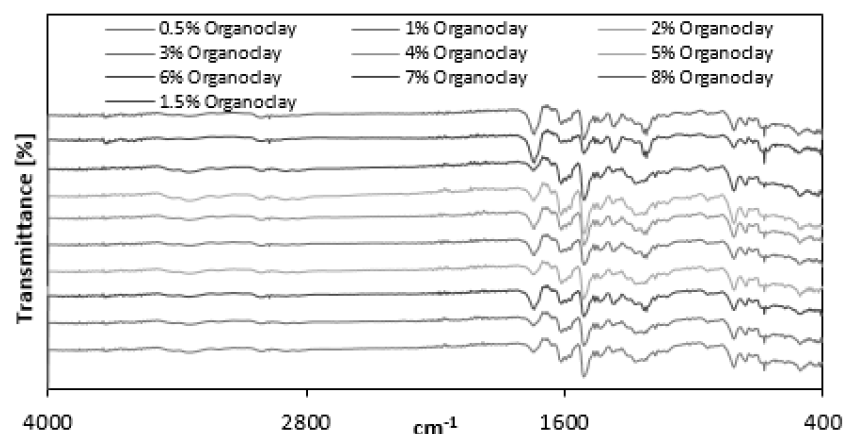

Fig. 2. FTIR spectra for organoclay-reinforced AF composites.

The characteristic peaks of primer amines as doublet at 3420 and $3350 \mathrm{~cm}^{-1}$ have disappeared in the 
diagrams of composites. Thus, two active protons of aniline have reacted with the formaldehyde. At 1600$1500 \mathrm{~cm}^{-1}$ there are peaks of aromatic $-\mathrm{C}=\mathrm{C}-$ bonds. At $1400-1300 \mathrm{~cm}^{-1}$, bands show existence of $-\mathrm{CH}_{2}-\mathrm{N}$ [17]. Stretching vibrations of aromatic protons result with peaks at $2996 \mathrm{~cm}^{-1}$ and out of plane bending vibrations at $650-750 \mathrm{~cm}^{-1}$. The bands in range of $1387-$ $1228 \mathrm{~cm}^{-1}$ are due to $\mathrm{C}-\mathrm{N}$ stretching (band between the aromatic ring and amino group) [3]. Peaks at 3027 and $2850 \mathrm{~cm}^{-1}$ are due to aromatic and aliphatic $\mathrm{C}-\mathrm{H}$ stretch, respectively. The $\mathrm{C}-\mathrm{H}$ stretching in formaldehyde generally comes at $2850 \mathrm{~cm}^{-1}$. It confirms that the presence of $-\mathrm{CH}_{2}$ moiety in $\mathrm{AF}$ has been introduced from formaldehyde unit [18].

\section{2. $X R D$ and SEM analysis}

XRD and SEM are frequently used to characterize morphology of nanocomposites. Figure 3 shows the XRD patterns for the composites. As seen from this figure, up to $4 \%$ of organo clay content, the organo clay platelets have been almost completely exfoliated in the polymer matrix. At high organo clay content, the characteristic smectite peak shifted to the left, indicating that the polymer chain is intercalated in the interlayer region of clay. It should be stressed here that the curing rate of the resin can also have a determining role in the intercalation process.

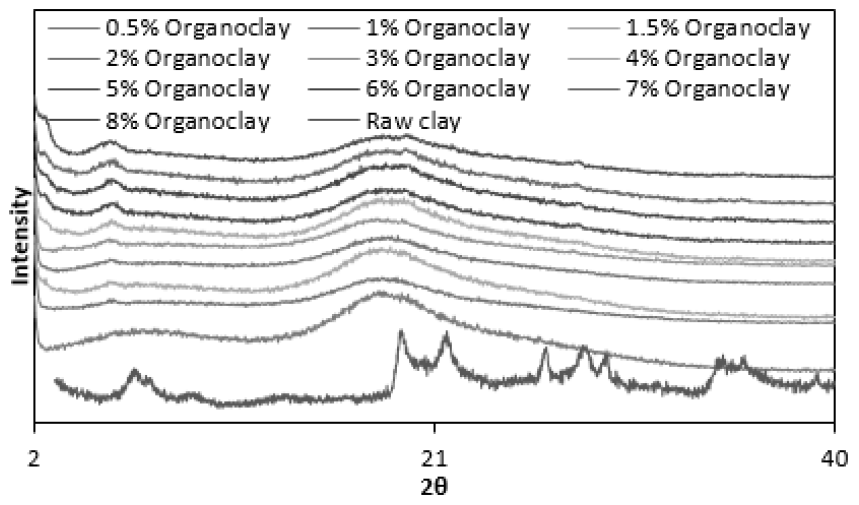

Fig. 3. The XRD patterns of organoclay reinforced AF composites and Na-Montmorillonite.

The SEM images of AF/organoclay composites prepared using various organoclay ratios are shown in Fig. 4.

This figure demonstrates that the surface morphologies of the composites have changed significantly with increasing organoclay content. The surface morphologies of the composites prepared with low organoclay contents (up to $4.0 \%$ ) reflect an almost full exfoliated dispersion of organoclay platelets in the polymer matrix. However, the surface morphologies obtained from the composites prepared with high contents of organoclay may imply that there is the intercalated-dispersion of the polymer chains in the interlayer region of the organoclay and also a significant reduction in the cross-linking intensity between
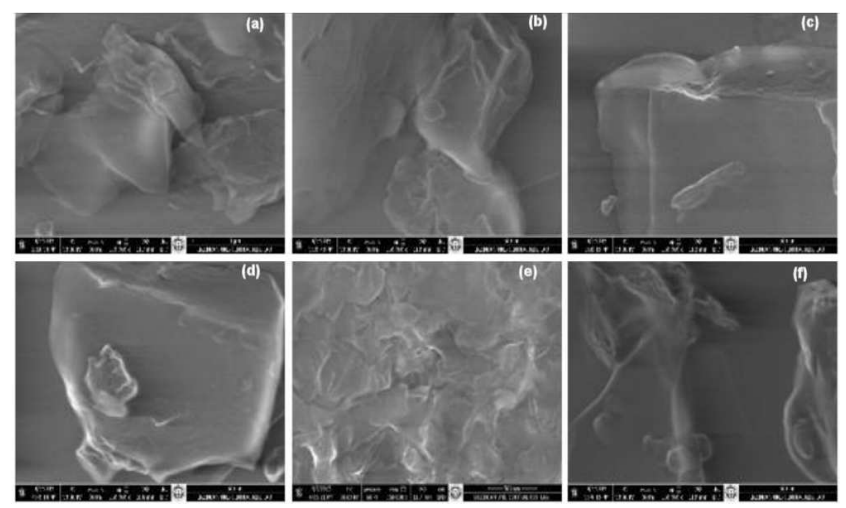

Fig. 4. The SEM images of the composites prepared using various organoclay ratios: 1 wt.\% (a), 2 wt.\% (b), 3 wt.\% (c), 4 wt.\% (d), 7 wt.\% (e), and 8 wt. \% (f).

the polymer chains. The major changes observed in the morphology may be due to the swelling which occurred as a result of the insertion of the reinforcing components into the polymer matrix [19]. The effect of $-\mathrm{CH}_{2}{ }^{-}$ linkages in the aniline-formaldehyde resin can be determined with the polymerization of aniline formaldehyde conducted under the acidic condition. Since any spherical texture in the composites prepared in this study was not observed, it can be concluded that the $-\mathrm{CH}_{2}-$ linkage plays a main role in the aniline sphere formation [20]. The extent of crystalline character depends on the acidic nature of the monomers [21].

\subsection{DSC analysis}

The characteristic temperature range of the DSC curves gives valuable information on the cure reaction of resins [22]. The DSC curves for the organoclay-reinforced AF composites are shown in Fig. 5.

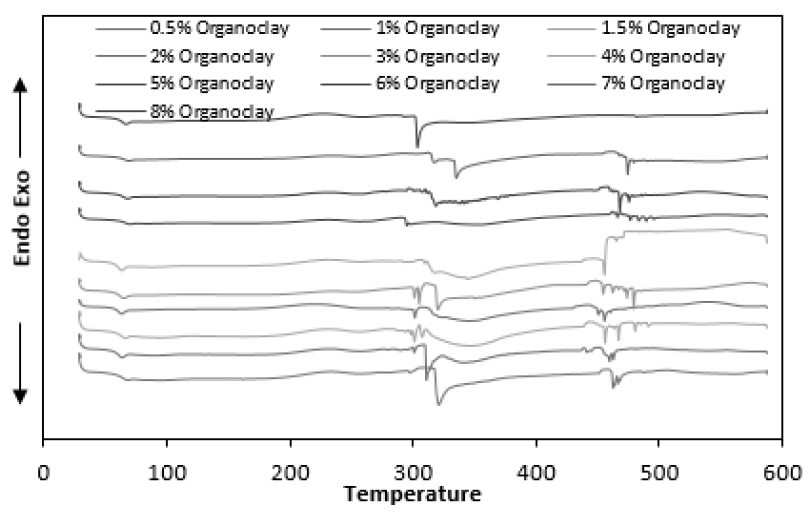

Fig. 5. The DSC thermograms of organoclayreinforced AF composites.

As can be seen from this figure, the glass transition temperature $\left(T_{\mathrm{g}}\right)$ and the melting temperature of the prepared composites with the increasing clay content are partially increased. Thermal decomposition characteristics of the composites at high temperatures exhibit 
a significant change, as well as, possibly, the higher clay content increases the thermal stability of the composite. It can be said that in all composites, polymer chains exhibited a conformational arrangement leading to an amorphous lattice.

\section{Conclusions}

- $\mathrm{AF} /$ organoclay composites have been synthesized using in situ intercalative polymerization technique with aniline formaldehyde resin (AF) and organoclay prepared using CTAB.

- The variation of thermal and textural characterization of $\mathrm{AF} /$ organoclay composites with increased clay ratio has been investigated by considering the DSC, XRD and FT-IR analyses.

- X-Ray diffractograms depict that $\mathrm{CTA}^{+}$ions intercalated from bulk into the interlayer region of the clay and $\mathrm{CTA}^{+}$ions adsorbed onto the clay platelets lead to a significant increase in the basal distance.

- In FTIR analysis, it was seen that the peak intensities of all composites increase with the increasing organoclays content. This may be explained by the better dispersion of clay particles in the matrix or steric interference to such interactions resulting from the increase in the amount of the clay.

- The DSC analysis has shown that organoclay causes a significantly improvement in thermal stability of the composite and the change of textural arrangement of the polymer chains during the polymerization.

\section{References}

[1] F. Mustaţá, I. Bicu, Polimery 47, 817 (2002).

[2] P.A. Kumar, M. Ray, S. Chakraborty, J. Hazard. Mater. 143, 24 (2007).

[3] S. Parveen, T. Ahamad, A. Malik, N. Nishat, Polym. Advan. Technol. 19, 1779 (2008).
[4] X. Lu, W. Zhang, C. Wang, T-C. Wen, Y. Wei, Prog. Polym. Sci. 36, 671 (2011).

[5] J.A. Yasmin, J. Luo, J.L. Abot, I.M. Daniel, Compos. Sci. Technol. 66, 2415 (2006).

[6] Ç. Doğar, A. Gürses, S. Köktepe, F. Mindivan, K. Günes, S. Aktürk, Acta Phys. Pol. A 125, 374 (2014).

[7] A. Gürses, S. Karagöz, F. Mindivan, K. Güneş C. Doğar, S. Aktürk, Acta Phys. Pol. A 125, 368 (2014).

[8] M. Alshabanat, A. Al-Arrash, W. Mekhamer, J. Nanomater. 2013, 1 (2013).

[9] B.N. Jang, C.A. Wilkie, Polymer 46, 2933 (2005).

[10] J. Golebiewski, A. Rozanski, J. Dzwonkowski, A. Galeski, Eur. Polym. J 44, 270 (2008).

[11] J. Zhang, R.K. Gupta, C.A. Wilkie, Polymer 47, 4537 (2006).

[12] Y.H. Yu, J.M. Yeh, S.J. Liou, C.L. Chen, D.J. Liaw, H.Y. Lu, J. App. Polym. Sci. 92, 3573 (2004).

[13] G.R. Saad, H.F. Naguib, S.A. Elmenyawy, J. Therm. Anal. Calorim. 111, 1409 (2012).

[14] L.B. de Paiva, A.R. Morales, R. Francisco, V. Díaz, Appl. Clay Sci. 42, 8 (2008).

[15] M.K. Wang, S.L. Wang, W.M. Wang, Soil Sci. Soc Am. J. 60, 138 (1996).

[16] M.J. Rosen, H.A. Goldsmith, Systematic Analysis of Surface-Active Agents, Wiley-Interscience, New York 1972.

[17] E. Ateş, N. Kızılcan, Pigm. Resin Technol. 40, 29 (2011).

[18] T. Maity, B.C. Samanta, S. Dalai, A.K. Banthi, Mater. Sci. Eng. A 464, 38 (2007).

[19] S.H. Hosseini, M. Malekdar, S. Naghdi, Polym. J. 42, 640 (2010)

[20] R.R. Koner, P.A. Kumar, S. Chakraborty, M. Ray, J. App. Polym. Sci. 110, 1158 (2008).

[21] W.B. Gurnule, S.S. Butoliya, J. App. Polym. Sci. 122, 2181 (2011).

[22] D. Fan, J. Li, J. Chang, Eur. Polymer J. 45, 2849 (2009). 